\title{
Comparação de três métodos para estimativa do índice de área foliar em pastos de capim-marandu sob lotação contínua
}

\author{
André Fischer Sbrissia ${ }^{1}$, Sila Carneiro da Silva ${ }^{2}$ \\ ${ }^{1}$ Programa de Pós-graduação em Agronomia - Escola Superior de Agricultura "Luiz de Queiroz" - Departamento de Zootecnia. \\ 2 Universidade de São Paulo - Escola Superior de Agricultura "Luiz de Queiroz" - Departamento de Zootecnia. Avenida Pádua Dias, 11 - \\ CEP: 13418-900, Caixa Postal 9, Piracicaba, SP, Brasil. Pesquisador do CNPq.
}

RESUMO - Objetivou-se comparar três métodos de determinação do IAF em pastos de capim-marandu (Brachiaria brizantha Hochst. ex A. Rich cv. Marandu) mantidos a quatro alturas de pastejo (10, 20, 30, e $40 \mathrm{~cm}$ ) por meio de lotação contínua com bovinos de corte. Foram comparados um método direto, destrutivo ( $\mathrm{IAF}_{\mathrm{MD}}$ ), um método indireto, destrutivo, baseado no uso da densidade populacional de perfilhos e área foliar média por perfilho (IAF ${ }_{\mathrm{DPP}}$ ), e um método indireto, não

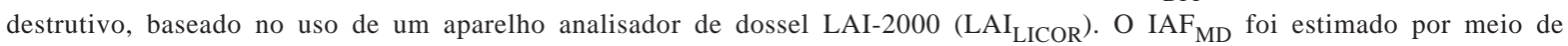
coletas de amostras de forragem contidas no interior de quatro retângulos de $0,11 \mathrm{~m}^{2}(0,30 \times 0,37 \mathrm{~m})$. As avaliações foram realizadas mensalmente e agrupadas em cinco épocas do ano: verão, outono, inverno, início e final de primavera. O experimento foi realizado segundo um delineamento de blocos completos casualizados, com quatro repetições, e arranjo de parcelas subdivididas, em que as alturas corresponderam às parcelas e os métodos, às subparcelas. Em comparação aos outros dois métodos, o analisador de dossel superestima os valores de IAF, provavelmente em virtude da grande deposição de material morto

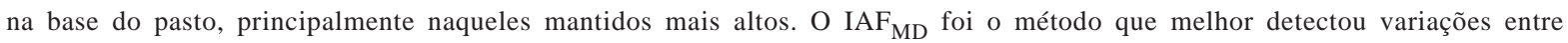
alturas e épocas do ano e o $\mathrm{IAF}_{\mathrm{DPP}}$ resultou em valores intermediários, com padrão de evolução nos valores absolutos similar ao registrado para o $\mathrm{IAF}_{\mathrm{MD}}$. $\mathrm{O}$ analisador de dossel não deve ser utilizado visando coletar dados de IAF para fins de simulação e modelagem matemática ou para estimar a eficiência de uso da radiação incidente.

Palavras-chave: altura do pasto, densidade populacional de perfilhos, IAF, LAI-2000

\section{Comparison of three methods for estimating leaf area index of marandu palisadegrass swards under continuous stocking}

\begin{abstract}
The objective of this research was to compare three methods for estimating LAI in marandu palisadegrass (Brachiaria brizantha Hochst. ex A. Rich cv. Marandu) swards subjected to four grazing heights (10, 20, 30 and $40 \mathrm{~cm}$ ) under continuous stocking by beef cattle. Evaluated methods were: (1) Direct, destructive ( $\mathrm{LAI}_{\mathrm{DM}}$ ); (2) Indirect, destructive, based on the use of tiller population density and mean leaf area per tiller $\left(\mathrm{LAI}_{\mathrm{TPD}}\right)$; and (3) Indirect, non-destructive, based on the use of a LAI-2000 canopy analyser equipment ( $\left.\mathrm{LAI}_{\mathrm{LICOR}}\right)$. $\mathrm{LAI}_{\mathrm{DM}}$ was estimated on herbage samples harvested using four $0.30 \times 0.37 \mathrm{~m}$ metallic frames per paddock. Measurements were carried out in a monthly basis and results grouped into five seasons of the year: summer, autumn, winter, early and late spring. The experiment was carried out according to a complete randomised block design, with four replications and a split-plot arrangement, where sward heights were allocated to plots and LAI estimation methods to sub-plots. The results showed that the canopy analyser overestimated LAI values in relation to the other two methods. This overestimation is mainly due to the excessive accumulation of dead material at the base of swards, particularly on those kept taller (30 and $40 \mathrm{~cm}$ ). The $\mathrm{LAI}_{\mathrm{DM}}$ was the method that better detected variations in sward height and seasons of the year, and the $\mathrm{LAI}_{\mathrm{TPD}}$ showed intermediate values, with a pattern of variation in absolute values similar to that obtained for the $\mathrm{LAI}_{\mathrm{DM}}$. The canopy analyser should not be used when LAI estimates are being generated for simulation and mathematical modelling purposes and to estimate the efficiency of incident radiation use.
\end{abstract}

Key Words: LAI-2000, LAI, sward height, tiller population density

\section{Introdução}

O índice de área foliar (IAF) pode ser definido como a relação entre a área foliar de uma das faces das folhas e a área de solo ocupada pelas folhas (Watson, 1947). Desde sua definição, o IAF passou a ser considerado uma variávelchave na compreensão da dinâmica da vegetação em ecossistemas terrestres, uma vez que é determinante de 
processos produtivos como a interceptação da luz pelo relvado e trocas gasosas e de água com o meio, além de interferir em aspectos ecológicos importantes, como a competição inter e intra-específica entre plantas, a retenção de carbono e a conservação do solo e ser um componente-chave dos ciclos biogeoquímicos em ecossistemas (Bréda, 2003).

O método clássico de determinação do IAF em culturas agrícolas consiste em medir a área foliar do relvado usando um planímetro (ou integradores de área) ou por meio de relações específicas entre determinada medida da folha (i.e., largura, comprimento etc.) e sua área. De modo geral, a área foliar é medida por meio de subamostras e relacionada à massa seca das folhas (por exemplo, via área foliar específica, AFE, $\mathrm{cm}^{2} \cdot \mathrm{g}^{-1}$ ). Dessa forma, a massa seca total de folhas coletadas em uma área conhecida de solo é convertida em IAF por meio de sua multiplicação com a AFE. Outra forma de determinação semelhante, porém não menos dispendiosa em termos de tempo e esforço, consiste em contar o número de perfilhos (ou plantas) em uma determinada área, selecionar alguns, e medir suas áreas foliares. $\mathrm{O}$ IAF, nesse caso, é obtido multiplicando-se a densidade populacional de perfilhos pela área foliar média por perfilho (Lemaire \& Chapman, 1996; Matthew et al., 2000). Esses dois métodos podem ser considerados destrutivos, pois exigem o corte de alguma área do pasto (ou de alguns perfilhos) para sua determinação.

Recentemente, têm surgido no mercado equipamentos eletrônicos que permitem inferir uma leitura indireta não destrutiva do IAF do dossel. O princípio básico de funcionamento desses equipamentos consiste em integrar variáveis como a transmissão e a penetração da luz e as características estruturais do relvado (ângulo e distribuição da folhagem). Nesse caso, o cálculo do IAF é realizado por meio de equações matemáticas (Bréda, 2003). Wilhelm et al. (2000) compararam três analisadores de dossel disponíveis no mercado e não constataram diferenças nas estimativas de IAF obtidas por esses três aparelhos em relação àquelas obtidas por meio de métodos destrutivos para a cultura do milho. Da mesma forma, Jesus et al. (2001) também não detectaram diferenças na determinação do IAF para a cultura do feijoeiro utilizando um aparelho analisador de dossel LAI 2000 (LI-COR LAI 2000, Lincoln NE, EUA) e relações empíricas obtidas a partir de medidas da largura das folhas.

Praticamente não existem trabalhos comparando métodos de estimativa do IAF em plantas forrageiras, particularmente aquelas de clima tropical. Desse modo, o objetivo neste trabalho foi comparar três métodos de determinação do IAF em pastos de capim-marandu (Brachiaria brizantha
Hochst. ex A. Rich cv. Marandu) mantidos a quatro alturas de pastejo por meio de lotação contínua com bovinos de corte: um método destrutivo direto $\left(\mathrm{IAF}_{\mathrm{MD}}\right)$; um método destrutivo indireto baseado na densidade populacional de perfilhos e na área foliar média por perfilho $\left(\mathrm{IAF}_{\mathrm{DPP}}\right)$; um método não-destrutivo indireto baseado no uso de um aparelho analisador de dossel LAI $2000\left(\mathrm{IAF}_{\text {LICOR }}\right)$.

\section{Material e Métodos}

O experimento foi conduzido na Unidade Experimental de Plantas Forrageiras (UEPF), em área do Departamento de Zootecnia da Escola Superior de Agricultura "Luiz de Queiroz", da Universidade de São Paulo, em Piracicaba, SP (22042' S, 47 $37^{\circ}$ W, $550 \mathrm{~m}$ altitude) em pastos de capimmarandu estabelecidos em setembro de 2000. O solo da área é um Nitossolo Vermelho eutroférrico, com horizonte A moderado e textura de argilosa a muito argilosa (EMBRAPA, 1999), de alta fertilidade. A análise de solo, realizada antes do período experimental, revelou os seguintes valores: $\mathrm{P}=$ $67 \mathrm{mg} / \mathrm{dm}^{3}$, resina trocadora de íons(Van Raij et al., 1986); $\mathrm{MO}=41 \mathrm{~g} / \mathrm{dm}^{3} ; \mathrm{pH}\left(\mathrm{CaCl}_{2}\right)=5,6 ; \mathrm{K}=6,5 \mathrm{mmol}_{\mathrm{c}} / \mathrm{dm}^{3} ; \mathrm{Ca}=$ $74 \mathrm{mmol} / \mathrm{dm}^{3} ; \mathrm{Mg}=19 \mathrm{mmol} / \mathrm{dm}^{3} ; \mathrm{H}+\mathrm{Al}=36 \mathrm{mmol}_{\mathrm{c}} / \mathrm{dm}^{3}$; $\mathrm{CTC}=135,0 \mathrm{mmol}_{\mathrm{c}} / \mathrm{dm}^{3}$; saturação de bases $=74 \%$. Em razão da elevada fertilidade, somente adubações nitrogenadas e, eventualmente, potássicas foram realizadas durante o período experimental (Tabela 1). Segundo o sistema Köppen, o clima da região de Piracicaba é classificado como Cwa, ou seja, mesotérmico úmido, subtropical de inverno seco (Figuras 1 e 2, respectivamente).

O experimento foi realizado segundo um delineamento de blocos completos casualizados, com quatro repetições e arranjo de parcelas subdivididas. As parcelas (piquetes de $1.200 \mathrm{~m} 2$ ) consistiram de quatro alturas de dossel forrageiro, 10, 20, 30 e $40 \mathrm{~cm}$, mantidas "constantes" por meio de lotação contínua e taxa de lotação variável com bovinos de corte. O monitoramento da altura dos pastos (Figura 3 ) foi realizado a intervalos de 3 e 4 dias ( 2 vezes por semana), em 20 pontos por unidade experimental, com o uso de transparência e régua (Fagundes et al., 1999; Pedreira, 2002). Foi estabelecida uma amplitude de variação de $10 \%$ para os valores de altura média dos tratamentos e, conforme o limite superior ou o inferior eram ultrapassados, os animais eram adicionados ou removidos das parcelas, respectivamente. Ao final do mês de outubro de 2001, todas as parcelas já se encontravam sob pastejo, assegurando um período de aproximadamente 60 dias de equilíbrio entre o estabelecimento das condições de pasto planejadas e o início das avaliações. O período experimental teve início em 
Tabela 1 - Datas, doses e tipos de fertilizantes utilizados duran te o período experimental

\begin{tabular}{lccc}
\hline Data & $\begin{array}{c}\text { Nitrogênio } \\
(\mathrm{kg} / \mathrm{ha})\end{array}$ & $\begin{array}{c}\text { Potássio } \\
\left(\mathrm{kg} / \mathrm{ha} \mathrm{de} \mathrm{K}_{2} \mathrm{O}\right)\end{array}$ & Fertilizante \\
\hline $7 / 11 / 2001$ & 66 & & Nitrato de amônio \\
$1 / 12 / 2001$ & 30 & 20 & 30.00 .20 \\
$10 / 1 / 2002$ & 45 & 30 & 30.00 .20 \\
$7 / 3 / 2002$ & 115 & & Uréia \\
$30 / 10 / 2002$ & 46 & & Uréia \\
Total & 302 & 50 & \\
\hline
\end{tabular}

8 de janeiro e terminou em 17 de dezembro de 2002, totalizando 343 dias.

Antes das avaliações de IAF pelos diferentes métodos, foram realizados pré-ensaios para determinação do número e tamanho de amostras a serem retiradas, de modo que o número de unidades amostrais que seriam utilizadas para compor as médias de cada piquete foi o resultado da melhor combinação possível entre o tempo gasto para sua realização

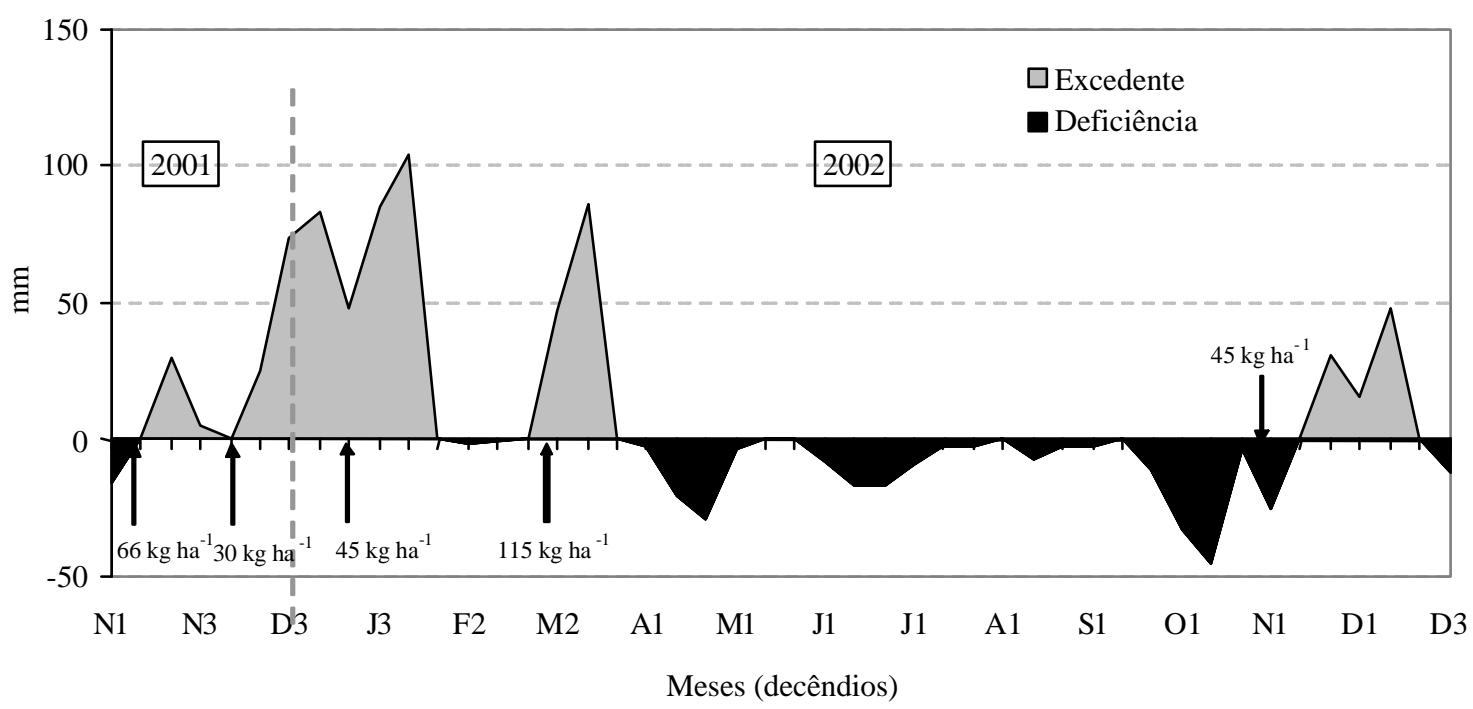

Figura 1 - Balanço hídrico dos decêndios mensais do período de novembro de 2001 a dezembro de 2002 e épocas de adubação nitrogenada. * Os símbolos no eixo X indicam as iniciais dos meses de cada ano. Desse modo, N1 corresponde ao primeiro decêndio do mês de novembro e assim sucessivamente.

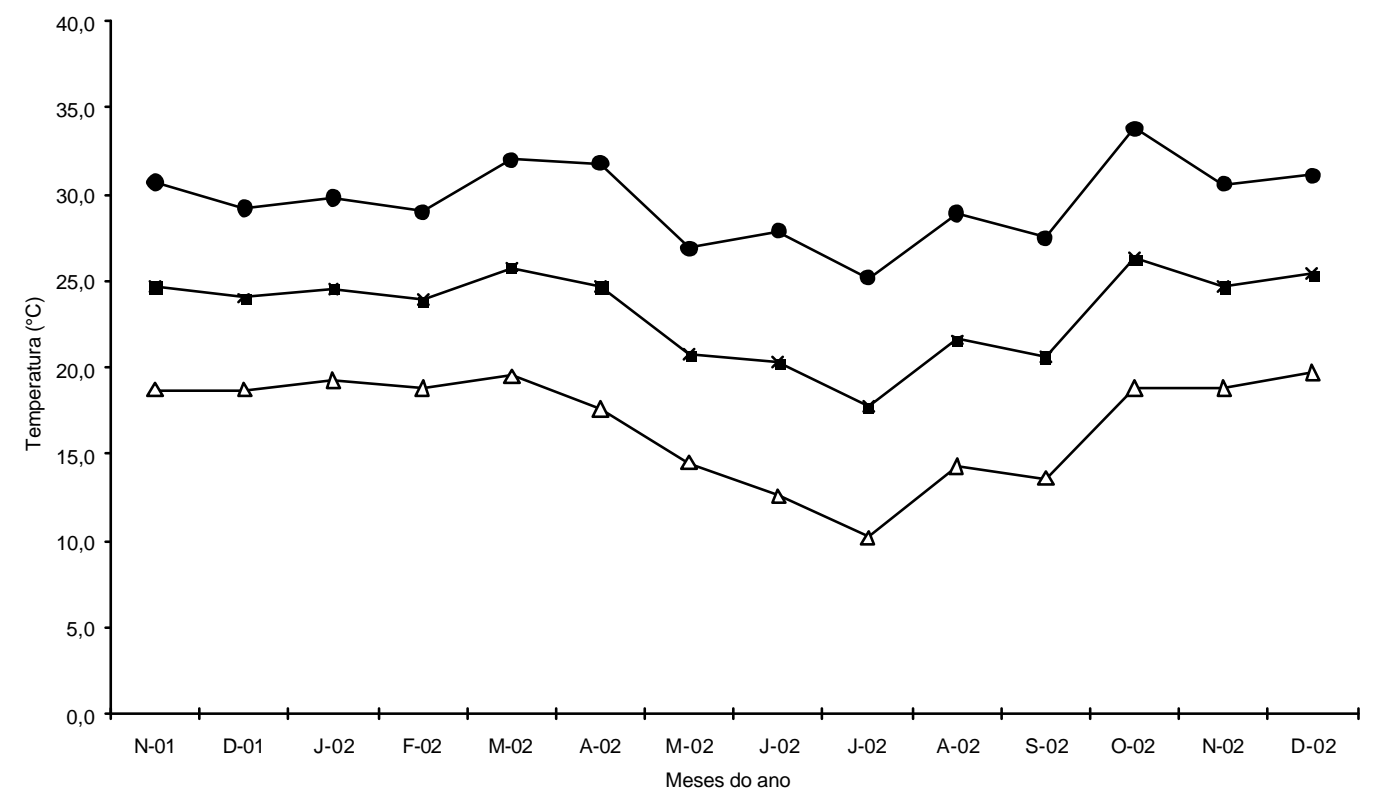

Figura 2 - Temperaturas médias $(\boldsymbol{\square})$, médias mínimas $(\Delta)$ e médias máximas $(\bullet)$ durante o período experimental. 
e o menor coeficiente de variação possível. Para avaliação do IAF pelo métododireto destrutivo, foram colhidas amostras de forragem contidas no interior de quatro retângulos de $0,11 \mathrm{~m}^{2}(0,30 \times 0,37 \mathrm{~m})$ por unidade experimental, respeitando-se um intervalo de quatro semanas entre amostragens sucessivas. A área amostrada correspondeu, sempre, a uma região do pasto que estivesse com uma altura média semelhante à da unidade experimental (piquete) no momento da amostragem. A forragem foi cortada no nível do solo, acondicionada em sacos plásticos e refrigerada a $4^{\circ} \mathrm{C}$. As amostras foram então lavadas e subamostradas, com o material restante colocado em estufa de ventilação forçada para secagem a $65^{\circ} \mathrm{C}$.

As subamostras foram fracionadas manualmente nos seguintes componentes: material morto (incluiu a parte morta/necrosada da folha senescente), invasoras, colmos (bainhas foliares e colmo) e folhas (lâminas foliares). Após a separação dos componentes morfológicos, as lâminas foliares tiveram suas áreas medidas em um integrador de área foliar LI-COR modelo LAI-3100 antes da secagem. Em seguida, esses componentes foram acondicionados em sacos de papel devidamente identificados e levados à estufa de ventilação forçada para secagem a $65^{\circ} \mathrm{C}$ até massa constante, quando foram pesados. Com o valor da massa seca de lâminas foliares da subamostra e da leitura de área foliar do aparelho, foi calculada a relação entre massa seca de lâminas foliares e área foliar. Essa relação foi utilizada para se determinar a área foliar da amostra da qual a subamostra foi retirada. Os valores de IAF foram calculados a partir do quociente entre a área foliar da amostra e a área correspondente de amostragem. As massas secas de lâminas foliares e de material morto foram convertidas de forma análoga para $\mathrm{kg}$ de $\mathrm{MS} / \mathrm{ha}$.

Matematicamente, o IAF de um pasto exclusivo de gramíneas é o produto de três características estruturais do dossel (Lemaire \& Chapman, 1996; Matthew et al., 2000):

$\mathrm{IAF}=$ densidade populacional de perfilhos $\times$ área média da folha $\times$ número de folhas vivas por perfilho.

Assim, para determinar o IAF por meio desta fórmula, avaliou-se a densidade populacional de perfilhos (DPP), bem como a área foliar média por perfilho (integrando os dois últimos fatores da equação acima). As estimativas de densidade populacional de perfilhos foram obtidas com o uso de armações metálicas de $0,25 \mathrm{~m}^{2}(100 \mathrm{~cm} \times 25 \mathrm{~cm}$ ) (Hay et al., 2000). Foram utilizados três retângulos por unidade experimental, os quais foram alocados na altura média dos pastos (altura) no momento da amostragem.Cadaavaliador ficou responsável pelas contagens em um bloco, de modo que eventuais diferenças entre observadores foram incluídas no efeito de bloco. As avaliações foram realizadas mensalmente e todos os perfilhos vivos contidos no interior da armação metálica foram contados.

Para a avaliação da área foliar média por perfilho, foram coletados 60 perfilhos por unidade experimental, em pontos representativos da altura média do dossel no momento da amostragem. Todos os perfilhos colhidos foram acondicionados em sacos plásticos e rapidamente levados à câmara fria para resfriamento $\left(4^{\circ} \mathrm{C}\right)$ e minimização dos efeitos de respiração e transpiração das plantas. Posteriormente, foram separados de cada perfilho as lâminas foliares verdes,

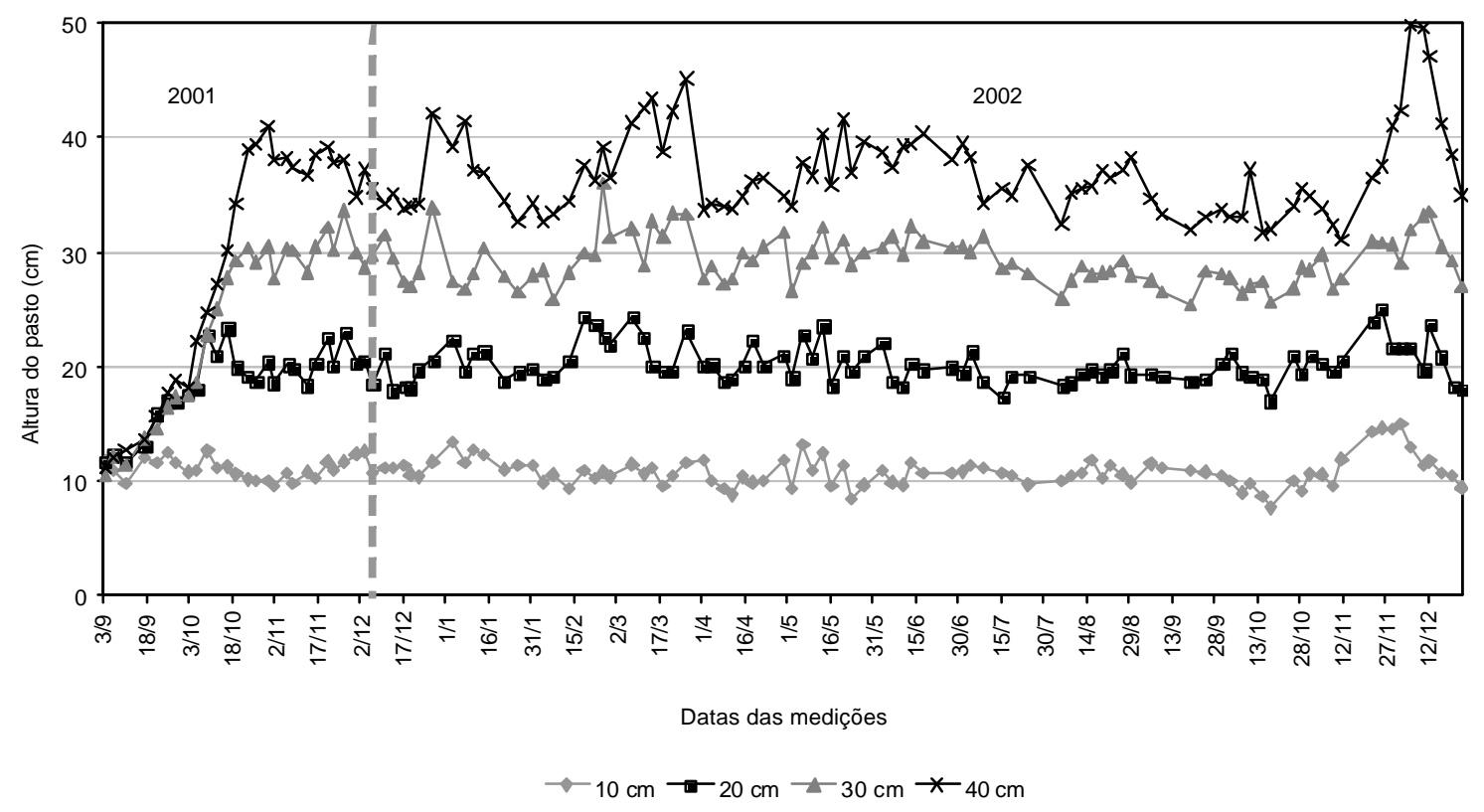

Figura 3 - Valores médios das alturas dos pastos ao longo do período experimental. 
o material morto e senescente e o colmo (colmo + bainha das folhas). A área foliar das lâminas verdes foi medida em aparelho integrador de área foliar, marca LI-COR, modelo LI3100, e consistiu do somatório das áreas foliares dos 60 perfilhos dividido por esse mesmo número. O IAF foi calculado multiplicando-se a densidade populacional de perfilhos pela área foliar média por perfilho.

$O$ índice de área foliar também foi estimado indiretamente com o aparelho analisador de dossel LI-COR modelo LAI 2000 (LI-COR, 1992). Esse aparelho é constituído de uma unidade de controle e um sensor em formato de barra articulada. O sensor é composto de um conjunto de lentes tipo olho de peixe que mede a radiação difusa simultaneamente em cinco bandas distintas sobre o ponto zenital. A imagem hemisférica é projetada para anéis, permitindo que cada um deles meça a radiação em cada banda chamada ângulo zênite. Um filtro ótico restringe a radiação transmitida abaixo de $490 \mathrm{~nm}$, minimizando a contribuição de luz dispersa pela folhagem. A unidade de controle recebe e registra os dados do sensor e executa os cálculos necessários para determinação do índice de área foliar e do ângulo médio de inclinação da folhagem. As medições são realizadas a partir de uma leitura de referência sobre o dossel forrageiro e uma ou mais leituras feitas abaixo do dossel (no nível do solo). As leituras abaixo do dossel são divididas pela leitura acima do dossel para obter a estimativa da luz incidente nos cinco ângulos zenitais. A recomendação é de que essas leituras sejam realizadas sob radiação difusa (céu encoberto, início da manhã ou final da tarde), pois assim evita-se uma superestimativa recorrente da elevada radiação transmitida pelas folhas quando a luz incide diretamente (Welles, 1990; Welles \& Norman, 1991; LI-COR, 1992). As medições foram realizadas mensalmente, sempre ao entardecer (LI-COR, 1992), em seis pontos representativos da condição média de cada unidade experimental no momento da amostragem. As medições consistiam de uma leitura acima e de cinco leituras abaixo do dossel forrageiro, realizadas no nível do solo, totalizando 6 leituras acima e 30 leituras abaixo do dossel por unidade experimental. A partir dessas leituras, o aparelho estimou o índice de área foliar dos pastos por meio de equações matemáticas (Welles \& Norman, 1991).

Os resultados foram inicialmente calculados com base nos meses do ano e plotados para visualização de possíveis padrões de variação ao longo do tempo. Análise visual dos dados permitiu a identificação de períodos em que o comportamento das variáveis estudadas era homogêneo, razão pela qual se optou por agrupar os valores mensais da seguinte forma: verão: janeiro, fevereiro e março; outono: abril, maio e junho; inverno: julho e agosto;

início de primavera: setembro e outubro; final de primavera: novembro e dezembro.

Optou-sepelo agrupamento dos dados de dois em dois meses a partir de julho com o objetivo de isolar os meses de setembro e outubro em um único período. Nesses dois meses, os pastos apresentaram padrões de resposta excepcionalmente diferentes dos demais, mas semelhantes entre si. Os dados, assim agrupados, foram analisados utilizando-se o procedimento MIXED (modelos mistos) do pacote estatístico SAS ${ }^{\circledR}$ (Statistical Analysis System) versão 8.2 para Windows ${ }^{\circledR}$. As médias entre tratamentos foram estimadas utilizando-se o LSMEANS e a comparação entre elas foi realizada por meio da probabilidade da diferença (PDIFF) usando o teste t de Student e nível de significância de $10 \%$.

As regressões lineares entre o $\mathrm{IAF}_{\mathrm{MD}}$ e o $\mathrm{IAF}_{\mathrm{DPP}} \mathrm{e}$ IAF $_{\text {LICOR }}$ e as correlações entre massa de folhas e material morto foram estimadas utilizando-se os programas GraphPad Prism versão 4.00 for Windows, GraphPad Software, San Diego Califórnia, USA e Past ${ }^{\circledR}$. Os coeficiente angulares das regressões foram estimados pelo método do "eixo maior reduzido" (do inglês, Reduced Major Axis, ou RMA), procedimento particularmente preferido nesse caso, no qual ocorre erro amostral nos dois eixos (LaBarbera, 1989).

A comparação entre os métodos foi realizada por meio de testes de paralelismo para regressões lineares entre os diferentes métodos avaliados (Stroppiana et al., 2006; Malone et al., 2002; Wilhelm et al., 2000). Brevemente, esse método testa a probabilidade de os coeficientes angulares das regressões serem iguais a 1 , ou seja, de que existe uma relação direta e significativa entre os métodos. Caso a probabilidade seja baixa $(\mathrm{P}<0,10)$, variações no IAF não seguem evolução proporcional e consistente entre os métodos avaliados.

\section{Resultados e Discussão}

Para os valores de IAF, foi detectado efeito de altura $(\mathrm{P}<0,0001)$, método de estimação $(\mathrm{P}<0,0001)$ e época do ano $(\mathrm{P}<0,0001)$, além das interações altura $\times$ método $(\mathrm{P}<0,0001)$, altura $\times$ época $(\mathrm{P}=0,003)$, método $\times$ época $(\mathrm{P}<0,0001)$ e altura $\times$ método $\times$ época $(\mathrm{P}<0,0001)$. Como o objetivo principal foi comparar as metodologias de estimação do IAF, são apresentados na Tabela 2 apenas os efeitos de altura, época e método de estimação e as interações altura $\times$ método e método $\times$ época.

Os valores de IAF dos pastos mantidos mais altos foram maiores que aqueles mantidos mais baixos (Figura 5), 
no entanto, houve diferenças significativas entre pastos mantidos a 30 e $40 \mathrm{~cm}$ apenas para o IAF $_{M D}$ (Tabela 2). Os maiores valores absolutos de IAF foram obtidos com o uso do aparelho analisador de dossel ( $\left.\mathrm{IAF}_{\mathrm{LICOR}}\right)$, de modo que $\mathrm{o} \mathrm{IAF}_{\mathrm{DPP}}$ apresentou valores intermediários àqueles obtidos com o LAI-2000 e com o método direto. A interação significativa entre altura $\times$ método sugere que os métodos utilizados detectaram de forma diferente variações em IAF para as alturas de pasto. $O$ método destrutivo $\left(\mathrm{IAF}_{\mathrm{MD}}\right)$ foi o único que mostrou diferenças de IAF entre as quatro alturas avaliadas, enquanto o método indireto $\mathrm{IAF}_{\mathrm{DPP}}$ não apresentou diferenças a partir da altura de $20 \mathrm{~cm}$, e aqueles obtidos por meio do analisador de dossel $\left(\mathrm{IAF}_{\mathrm{LICOR}}\right)$ não apresentaram diferenças a partir da altura de $30 \mathrm{~cm}$. O $\mathrm{IAF}_{\text {LICOR- }}$ foi maior que os demais métodos nas alturas de 30 e $40 \mathrm{~cm}$, semelhante ao $\operatorname{IAF}_{\mathrm{DPP}}$ na altura de $20 \mathrm{~cm}$ e ao $\mathrm{IAF}_{\mathrm{MD}}$ na altura de $10 \mathrm{~cm}$. Adicionalmente, para a altura de

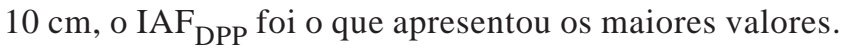

A estimativa do IAF por meio da densidade populacional de perfilhos (DPP) e da medição da área foliar por perfilho (AFP) integra duas variáveis que são medidas de forma independente e, portanto, com maior probabilidade de ocorrência de erros.

Assim, quando se coletam perfilhos para determinar suas áreas foliares, é normal descartar perfilhos sem folhas, o que resulta em superestimativa da área foliar média por perfilho, principalmente em pastos mantidos mais baixos, sujeitos às maiores intensidades de desfolhação.

De fato, os maiores valores (em relação ao $\mathrm{IAF}_{\mathrm{MD}} \mathrm{e}$ $\mathrm{IAF}_{\text {LICOR }}$ ) obtidos por este método foram observados em pastos mantidos a $10 \mathrm{~cm}$ (Tabela 2). No entanto, em alturas de dossel maiores, o IAF ${ }_{D P P}$ foi semelhante ao $\mathrm{IAF}_{\mathrm{MD}}$, uma vez que os perfilhos coletados representaram uma amostragem mais fiel da população de perfilhos existentes nos pastos.

A interação método de estimação $\times$ época do ano sugere também diferenças importantes na capacidade dos métodos em detectar variações sazonais em IAF. Variações sazonais foram detectadas com o uso do $\mathrm{IAF}_{\mathrm{MD}}$, enquanto que o IAF $F_{D P P}$ sugeriu não haver variações entre as épocas de inverno, início e final de primavera, que foram diferentes apenas dos períodos de verão e outono, enquanto o IAF $_{\text {LICOR }}$ mostrou diferenças apenas entre o verão e as demais épocas (Tabela 2).

O sensor do aparelho analisador de dossel LAI-2000 é incapaz de distinguir tecidos vivos de mortos (Welles \& Norman, 1991). Não distingue também folhas de outras partes da planta, como colmos (hastes) e pecíolos (Malone et al., 2002). Assim, toda a superfície da planta passível de contribuir para a interceptação de luz é considerada nos cálculos realizados pelo aparelho como área foliar. No caso de estimativas de IAF em pastos sob lotação contínua, a superestimativa dos dados com o uso do LAI-2000 pode também estar relacionada ao excessivo acúmulo de material morto na base do dossel (Molan, 2004).

O índice de área foliar obtido com os três métodos (Figura 4) esteve positivamente correlacionado à massa de folhas do pasto e o maior coeficiente de correlação foi verificado com o uso do método destrutivo direto $\left(\mathrm{IAF}_{\mathrm{MD}--}\right)$. Isso era esperado, uma vez que o uso dessa metodologia pressupõe relação direta entre a massa de folhas e sua área foliar específica. Apesar disso, a correlação entre os outros dois métodos foi significativa, indicando que, mesmo obtidos de forma independente, o IAF $_{\text {DPP }}$ e o IAF LICOR $_{\text {se }}$ correlacionaram significativamente com a massa de folhas. Por outro lado, a correlação entre o índice de área foliar e a massa de material morto dos pastos foi significativa apenas para o $\mathrm{IAF}_{\mathrm{LICOR}}$ e indica que a presença desse componente morfológico na massa de forragem do dossel poderia estar inflacionando os valores de IAF obtidos com esse método, sobretudo durante o inverno e o início da primavera (Tabela 2), quando a proporção de material morto nos pastos foi a mais alta de todo o período experimental (Molan, 2004).

O manual do fabricante sugere que pode haver divergências nas estimativas de IAF quando as plantas apresentam grande quantidade de material morto (Licor, 1992). Apesar de a proporção (\%) de material morto na massa de forragem dos pastos mantidos nas quatro alturas avaliadas ter sido semelhante (Molan, 2004), as quantidades de massa estimada em $\mathrm{kg}$ de MS/ha foram 3 a 4 vezes maiores nos pastos mantidos a $40 \mathrm{~cm}$ quando comparados àqueles mantidos a $10 \mathrm{~cm}$ (Molan, 2004). Portanto, o problema de superestimativa dos valores do IAF $_{\text {LICOR }}$ pode ser mais pronunciado em pastos mantidos mais altos.

Van Wijk \& Williams (2005) alertaram sobre as limitações do LAI-2000 em estimar o IAF em vegetações muito rasteiras, como aquelas de regiões árticas. Assim, era possível que, em pastos mantidos a $10 \mathrm{~cm}$, houvesse subestimativa nos valores de IAF, o que não ocorreu. No momento da medição, o sensor do LAI-2000 fica posicionado a aproximadamente $3 \mathrm{~cm}$ do solo. Isso significa que, em pastos mantidos a $10 \mathrm{~cm}$ de altura, o aparelho detectou aproximadamente $70 \%$ da superfície de interceptação de luz do dossel. No outro extremo, em pastos mantidos a $40 \mathrm{~cm}$, aproximadamente $93 \%$ da superfície de interceptação de luz foi considerada para estimativa do IAF. Isso porque, em pastos mais baixos, a maior concentração de material morto 
Tabela 2 - Índice de área foliar estimado por meio de três métodos em pastos de capim-marandu

\begin{tabular}{lcccc}
\hline & \multicolumn{4}{c}{ Método de estimação ${ }^{1}$} \\
\cline { 2 - 4 } & $\mathrm{IAF}_{\mathrm{MD}}$ & $\mathrm{IAF}_{\mathrm{DPP}}$ & $\mathrm{IAF}_{\mathrm{LICOR}}$ & Média \\
\hline Altura $(\mathrm{cm})$ & $1,7 \mathrm{Db}$ & $2,7 \mathrm{Ba}$ & $1,9 \mathrm{Cb}$ & $2,1 \mathrm{C}$ \\
10 & $3,1 \mathrm{Cb}$ & $5,0 \mathrm{Aa}$ & $5,6 \mathrm{Ba}$ & $4,6 \mathrm{~B}$ \\
20 & $3,8 \mathrm{Bc}$ & $5,0 \mathrm{Ab}$ & $6,6 \mathrm{Aa}$ & 5,1 \\
30 & $4,7 \mathrm{Ab}$ & $4,8 \mathrm{Ab}$ & $6,9 \mathrm{Aa}$ & $5,5 \mathrm{~A}$ \\
40 & \multicolumn{4}{c}{ e.p.d. da interação $=0,36$} \\
Média & $3,3 \mathrm{c}$ & $4,4 \mathrm{~b}$ & $5,3 \mathrm{a}$ & e.p.d ${ }^{*}=0,21$ \\
\hline
\end{tabular}

Época do ano

$\begin{array}{lllll}\text { Verão } & 5,1^{\mathrm{Aa}} & 5,4^{\mathrm{Aa}} & 4,5^{\mathrm{Bb}} & 5,0^{\mathrm{A}} \\ \text { Outono } & 3,6^{\mathrm{Bb}} & 5,3^{\mathrm{Aa}} & 5,2^{\mathrm{Aa}} & 4,7^{\mathrm{A}} \\ \text { Inverno } & 3,2^{\mathrm{BCb}} & 3,6^{\mathrm{Bb}} & 5,6^{\mathrm{Aa}} & 4,1^{\mathrm{B}} \\ \text { Início da primavera } & 2,0^{\mathrm{Dc}} & 3,7^{\mathrm{Bb}} & 5,4^{\mathrm{Aa}} & 3,7^{\mathrm{C}} \\ \text { Final da primavera } & 2,6^{\mathrm{CDc}} & 4,0^{\mathrm{Bb}} & 5,7^{\mathrm{Aa}} & 4,1^{\mathrm{BC}}\end{array}$

e.p.d. da interação $=0,39$

${ }_{1}^{1} \mathrm{~A} \mathrm{~F}_{\mathrm{MD}}=$ índice de área foliar obtido pelo método direto destrutivo, $I A F_{D P P}=$ índice de área foliar obtido pelo método indireto destrutivo e $I A F_{L I C O R}=$ índice de área foliar obtido pelo método indireto não-destrutivo. e.p.d. = erro-padrão da diferença.

Médias seguidas da mesma letra minúscula na linha e maiúscula na coluna não diferem entre si $(P>0,10)$.
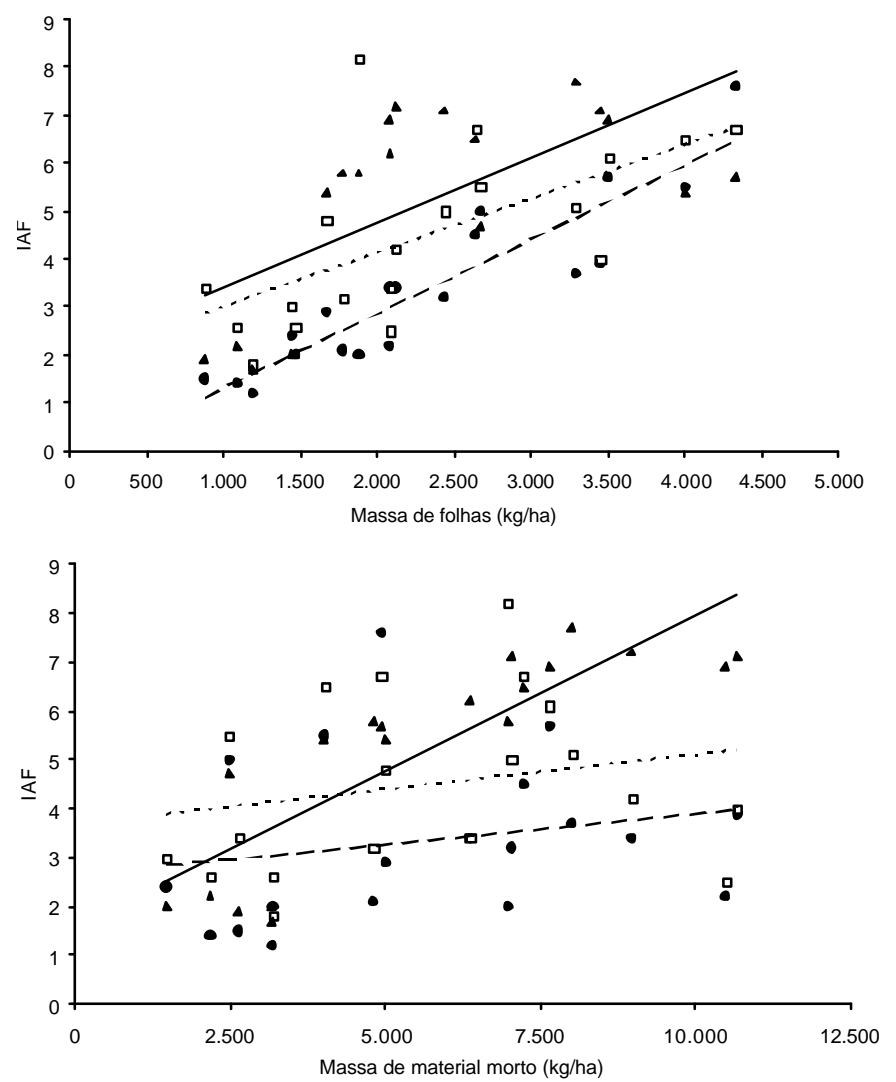

Figura 4 - Correlação entre os $I A F_{S}$ obtidos com os três métodos e a massa de folhas do pasto; $\mid \mathrm{AF}_{\mathrm{MD}}(\bullet, r=0,91$, $P<0,0001), I_{D P P}(\square, r=0,63, P=0,0035)$ e IAF

$(\mathbf{\Delta}), r=0,64, P=0,029)$ e com a massa de material morto do pasto; $I_{A F}(\bullet, r=0,20, P=0,41), I_{M D}$ $(\square, r=0,22, P=0,35)$ e IAPICOR $(\boldsymbol{\Delta}, r=0,85$, $P<0,0001)$. ocorreu nos primeiros $4 \mathrm{~cm}$ do dossel (Molan, 2004) e diminuiu progressivamente a partir dessa altura. Nas alturas maiores, no entanto, ocorreu o inverso, ou seja, houve aumento na proporção e na quantidade de material morto a partir de alturas superiores a $4 \mathrm{~cm}$.

A análise de regressão linear entre o $\mathrm{IAF}_{\mathrm{MD}} \mathrm{e}$ os outros dois métodos sugere que o $\mathrm{IAF}_{\mathrm{DPP}}$ apresentou padrão de evolução nos valores de IAF semelhante àquele verificado para o método direto destrutivo ( IAF $_{M D}$ ) (Figura 5, Tabela 3).

Para o IAF $\mathrm{DPP}_{\mathrm{DP}}$, a probabilidade de coeficientes angulares diferentes de 1 foi significativa apenas durante o início de primavera (Tabela 3), ou seja, apesar de as estimativas de IAF terem sido diferentes em relação àquelas obtidas a partir do método direto destrutivo, existiu uma relação linear entre $\mathrm{IAF}_{\mathrm{MD}}$ e $\mathrm{IAF}_{\mathrm{DPP}}$ na maior parte dos períodos avaliados. A regressão entre $\mathrm{IAF}_{\mathrm{MD}}$ e $\mathrm{IAF}_{\mathrm{LICOR}}$, por sua vez, comprovou que apenas durante o outono houve relação linear entre as estimativas de IAF obtidas por meio do analisador de dossel e o método direto destrutivo, sugerindo que, além de os valores absolutos serem diferentes, as variações nas observações entre as alturas e épocas do ano obedeceram a modelos matemáticos distintos entre o

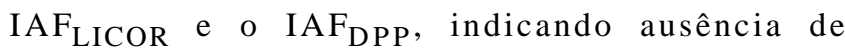
proporcionalidade constante (consistência) entre as estimativas. Ressalta-se que, a partir do período de outono, os coeficientes angulares obtidos para a regressão linear entre

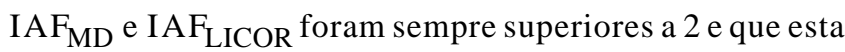
maior inclinação foi ocasionada principalmente peloselevados valores de IAF obtidos pelo LAI-2000 em pastos de $20 \mathrm{~cm}$ e, especialmente, naqueles mantidos a 30 e $40 \mathrm{~cm}$, justamente as situações em que houve o maior acúmulo de material morto na base dos pastos (Molan, 2004).

Com base nessas limitações, alguns autores têm sugerido que o IAF medido por meio de analisadores de dossel reflete, na verdade, outro tipo de índice, que tem recebido denominações variadas, como índice de área da planta (IAP), índice de área de interceptação da luz (IAIL) (Bréda, 2003) e índice de área da folhagem (IAFf) (Welles \& Norman, 1991; Molan, 2004).

Tabela 3 - Teste de paralelismo de retas (probabilidade de coeficientes angulares iguais a 1) para as regressões lineares entre IAF $F_{M D}$ e IAF $F_{D P P}$ e IAF $F_{\text {LICOR }}$ apresentadas na Figura 5

\begin{tabular}{lcc}
\hline Época do ano & $\mathrm{IAF}_{\mathrm{DPP}}$ & $\mathrm{IAF}_{\text {LICOR }}$ \\
\hline Verão & 0,298 & 0,001 \\
Outono & 0,491 & 0,380 \\
Inverno & 0,436 & 0,001 \\
Início de primavera & 0,022 & 0,001 \\
Final de primavera & 0,175 & 0,002 \\
\hline
\end{tabular}

() 2008 Sociedade Brasileira de Zootecnia 

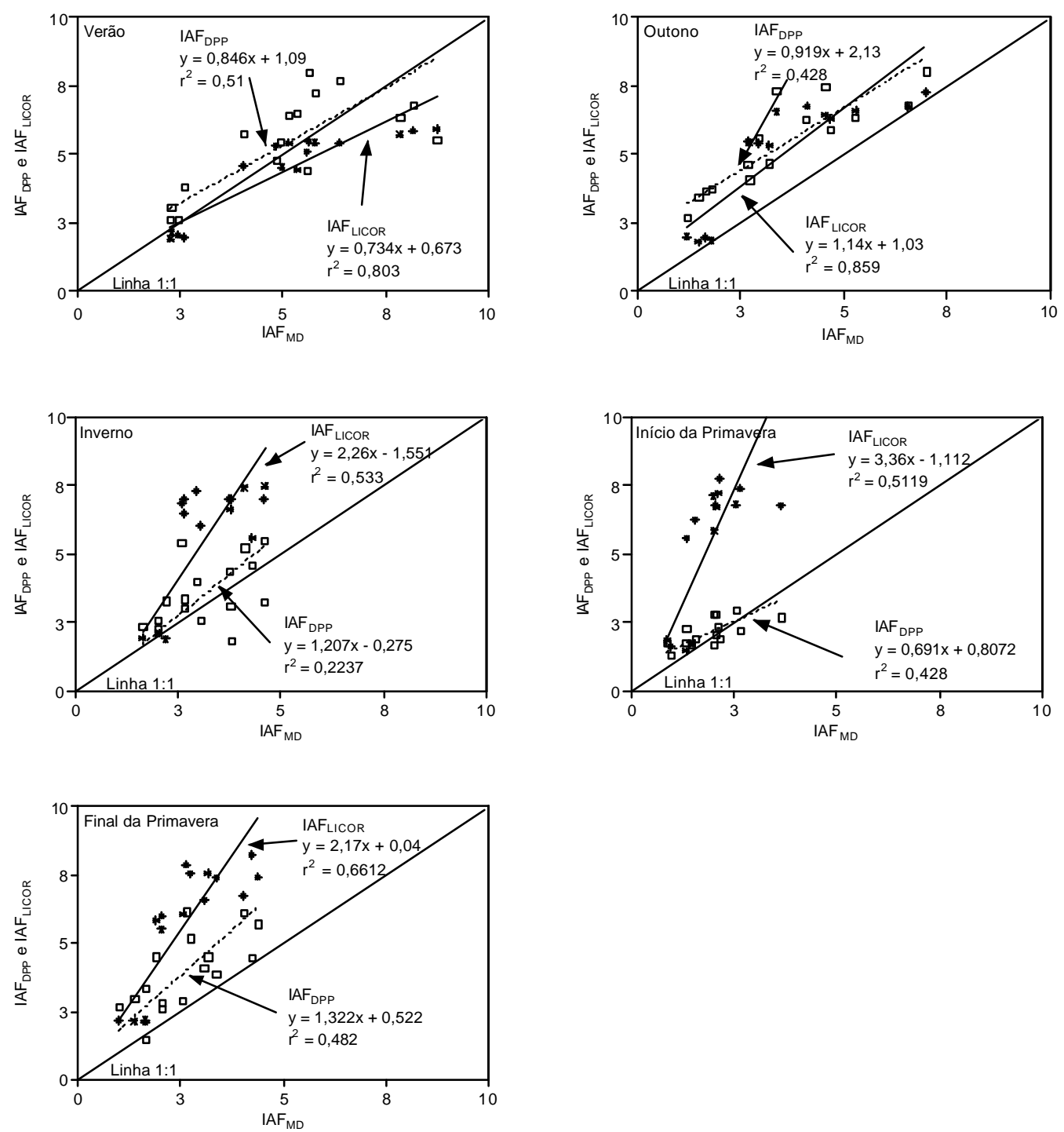

Figura 5 - Relação funcional entre o IAF $\mathrm{MD}_{\mathrm{MD}}$ e o IAF $\mathrm{DPP}_{\mathrm{DP}}$ e IAF $\mathrm{LIICR}_{\mathrm{C}}$ em diferentes épocas do ano.

O aparelho LAI-2000 permite que sejam feitas calibrações por meio de determinações diretas do IAF (LI-COR, 1992). Assim, teoricamente, seria possível gerar coeficientes de ajuste a fim de minimizar as diferenças de estimativas entre os métodos de leitura direta e indireta. No entanto, a variação sazonal na deposição e decomposição de material morto no dossel faria com que eventuais calibrações tivessem que ser feitas periodicamente, oque, na prática, seriainjustificável, uma vezque determinações diretas já forneceriam os valores de IAF.

\section{Conclusões}

Os valores de IAF gerados a partir de estimativas do aparelho analisador de dossel LAI-2000 em pastos de capim-marandu mantidos foram superestimados em relação aos valores obtidos por meio de métodos diretos destrutivos. O método baseado na multiplicação entre a densidade populacional de perfilhos e a área foliar média por perfilho mostrou-se relativamente adequado, porém, cuidados de vem ser tomados na amostragem dos perfilhos, principalmente em dosséis mantidos baixos e com uma proporção grande de perfilhos desfolhados.

\section{Agradecimento}

À FAPESP, pela concessão da bolsa de estudos; e aos membros da equipe de trabalho: Adriano Vecchiatti Lupinacci, Alexandre Campos Gonçalves, Daniel Oliveira de Lucena Sarmento, Leonardo Kehdi Molan e Flávia Maria Erbetta de Andrade. 


\section{Literatura Citada}

BRÉDA, N.J.J. Ground-based measurements of leaf area index: a review of methods, instruments and current controversies. Journal of Experimental Botany, v.54, n.392, p.2403$2417,2003$.

EMPRESA BRASILEIRA DE PESQUISA AGROPECUÁRIA EMBRAPA. Sistema brasileiro de classificação de solos. Brasília: 1999. 412p. (Produção de Informação).

FAGUNDES, J.L.; Da SILVA, S.C.; PEDREIRA, C.G.S. et al.Índice de área foliar, interceptação luminosa e acúmulo de forragem em pastagens de Cynodon spp. sob diferentes intensidades de pastejo. Scientia Agricola, v.56, n.4, p.1141-1150, 1999.

HAY, M.J.M.; JONES, R.M.; ORR, D.M. Plant population dynamics in grasslands. In: 'T MANNETJE, L.; JONES, R.M. (Eds.)Field and laboratory methods for grassland and animal production research. 1.ed. Cambridge: University Press, 2000. p.123-149.

JESUS, W.C.; VALE, F.X.R.; COELHO, R.R. et al. Comparison of two methods for estimating leaf area index on common bean. Agronomy Journal, v.93, n.5, p.989-991, 2001.

LABARBERA, M. Analyzing body size as a factor in ecology and evolution. Annual Review of Ecology and Systematics, v.20, p.97-117, 1989.

LEMAIRE, G.; CHAPMAN, D. Tissue flows in grazed plant communities. In: HODGSON, J.; ILLIUS A.W. (Eds.). The ecology and management of grazing systems. London: CAB International, 1996. p.3-36.

LEVY, P.E.; JARVIS, P.G. Direct and indirect measurements of LAI in millet and fallow vegetation in HAPEX-Sahel.Agricultural and Forest Meteorology, v.97, n.3, p.199-212, 1999.

LI-COR. Plant canopy analyzer: LAI-2000; operating manual. Lincoln: 1992. 179p.

MALONE, S.; HERBERT, D.A.; HOLSHOUSER, D.L. Evaluation of the LAI-2000 plant canopy analyzer to estimate leaf area in manually defoliated soybean. Agronomy Journal, v.94, n.5, p.1012-1019, 2002.
MATTHEW, C.; ASSUERO, S.G.; BLACK, C.K. et al. Tiller dynamics of grazed swards. In: LEMAIRE, G.; HODGSON, J.; MORAES, A. et al. (Eds.) Grassland ecophysiology and grazind ecology. Wallingford: CABI Publishing, 2000. p. $127-150$.

MOLAN, L.K. Estrutura do dossel, interceptação luminosa e acúmulo de forragem em pastos de capim-Marandu submetidos a alturas de pastejo por meio de lotação contínua. Piracicaba: Escola Superior de Agricultura Luiz de Queiroz, 2004. 159p. Dissertação (Mestrado em Agronomia Ciência Animal e Pastagens) - Escola Superior de Agricultura Luiz de Queiroz, 2004.

PEDREIRA, C.G.S. Avanços metodológicos na avaliação de pastagens. In: REUNIÃO ANUAL DA SOCIEDADE BRASIEIRA DE ZOOTECNIA, 39., 2002, Recife. Anais... Recife: Sociedade Brasileira de Zootecnia, 2002. p.100-150.

STROPPIANA, D.; BOSCHETTI, M.; CONFALONIERI, R. et al. Evaluation of LAI-2000 for leaf area index monitoring in paddy rice. Field Crops Research, v.99, n.2-3, p.167-170, 2006.

Van RAIJ, B.; QUAGGIO, J.A.; SILVA, N.M. Extraction of phosphorus, potassium, calcium, and magnesium from soils by ion-exchange resin procedure. Communications in Soil Science and Plant Analysis, v.17, n.5, p.547-566, 1986.

Van WIJK, M.T.; WILLIAMS, M. Optical instruments for measuring leaf area index in low vegetation: application in arctic ecosystems. Ecological Applications, v.15, n.4, p.1462-1470, 2005.

WATSON, D.J. Comparative physiological studies on the growth of field crops: I. Variation in net assimilation rate and leaf area between species and varieties, and with and between years. Annals of Botany, v.11, p.41-76, 1947.

WELLES, J.M. Some indirect methods of estimating canopy structure. Remote Sensing Reviews, v.5, n.1, p.31-43, 1990.

WELLES, J.M.; NORMAN, J.M. Instrument for indirect measurement of canopy architecture. Agronomy Journal, v.83, n.5, p.818-825, 1991.

WILHELM, W.W.; RUWE, K.; SCHLEMMER, M.R. Comparison of three leaf area index meters in a corn canopy. Crop Science, v.40, n.4, p.1179-1183, 2000. 